\title{
Silent atrial fibrillation episodes and cognitive impairment in patients with cardiac implantable devices. Protocol of the SAFE-COG ongoing observational single-center non-experimental case-control clinical study
}

\author{
Stefan Sawicki ${ }^{1}$, Anna Mierzyńska ${ }^{2}$, Agnieszka Krauze ${ }^{3}$, Piotr Dobrowolski ${ }^{4}$, Ewa Świerżyńska ${ }^{5}$, \\ Aleksander Prejbisz ${ }^{4}$, Piotr Michałek ${ }^{1}$, Maria Bilińska ${ }^{5}$, Anna Lutyńska ${ }^{6}$, Andrzej Januszewicz ${ }^{4}$, Maciej Sterliński ${ }^{5}$
}

'Rapid Diagnosis Department, National Institute of Cardiology, Warszawa, Poland 2Department of Rehabilitation, Medical Center of Postgraduate Education, Gruca Orthopedic and Trauma Teaching Hospital, Warszawa, Poland ${ }^{3}$ Department of Coronary Artery Disease and Cardiac Rehabilitation, National Institute of Cardiology, Warszawa, Poland ${ }^{4}$ Department of Hypertension, National Institute of Cardiology, Warszawa, Poland ${ }^{5} 1^{\text {st }}$ Department of Arrhythmia, National Institute of Cardiology, Warszawa, Poland ${ }^{6}$ Department of Medical Biology, National Institute of Cardiology, Warszawa, Poland

\section{Correspondence to:}

Stefan Sawicki, MD

Rapid Diagnosis Department,

National Institute of Cardiology,

Alpejska 42, 04-628 Warszawa,

Poland,

phone: +48223434238,

e-mail: ssawicki@ikard.pl

Copyright by the Author(s), 2021

Kardiol Pol. 2021;

79 (12): 1362-1364;

DOI: 10.33963/KP.a2021.0162

Received:

September 30, 2021

Revision accepted:

November 28, 2021

Published online:

November 29, 2021

\section{INTRODUCTION}

Atrial fibrillation (AF) is highly prevalent among the elderly and is associated with a higher risk of dementia. Silent Atrial Fibrillation Episodes and COGnitive impairment in patients with cardiac implantable devices (SAFE-COG) is an observational clinical trial that aims to assess the relationship between atrial high rate episodes (AHRE), a silent form of short, undiagnosed AF episodes revealed on cardiac implantable device interrogation, and cognitive impairment.

\section{METHODS}

SAFE-COG is an ongoing observational single-center non-experimental case-control clinical study. The study protocol was approved by the bioethics committee of the National Institute of Cardiology in Warsaw (IK-NPIA-0021-78/1800/2019).

We will compare two groups of 50 patients with previously implanted pacemakers or car- dioverter-defibrillators that can detect AHRE and record an intracardiac electrocardiogram (EGM). Both groups are matched according to age, sex, educational level, and history of hypertension. Inclusion and exclusion criteria are shown in Table 1. We set the group size at 100 individuals according to the results of previous studies and characteristics of methodology. The use of sensitive diagnostic methods detecting cognitive impairment and implantable device data excluding patients with undetected silent AF from the control group enabled us to set a study group with a lower number of subjects than previous studies [1, 2].

The decision to introduce oral anticoagulation (OAC) will be taken according to the guidelines and the patient's risk profile.

The groups will be evaluated in terms of body weight, height, history of myocardial infarction, hypercholesterolemia, diabetes, and nicotine addiction. Both groups will be

Table 1. Inclusion and exclusion criteria 
examined for thyroid dysfunction and primary hyperaldosteronism. Transthoracic echocardiography with particular emphasis on left atrium morphology will be performed. We will evaluate cognitive functions at the time of inclusion and after an 18-month observation period to estimate the general level of cognitive functioning and cognitive performance in the following areas: attention and executive functions, verbal memory, language functions, and visual-spatial functions. We will use the following neuropsychological questionnaires: Addenbrooke's Cognitive Examination III (J Hodges), the California Verbal Learning Test (DC Delis), and the Color Trails Test (LF D'Elia).

The risk of obstructive sleep apnea (OSAS) will be assessed using the Berlin Questionnaire.

Division into groups will be based on the presence of AHRE with a frequency of at least $180 \mathrm{bpm}$ and lasting at least 6 minutes, visually confirmed to be AF by EGM.

\section{Endpoints of the study}

1. The assessment-based comparison of the prevalence and severity of cognitive impairment in patients with and without AHRE.

2. The evaluation of the clinical characteristics of patients with AHRE, particularly the factors that may affect the occurrence of AHRE: left atrial and left ventricular remodeling and dysfunction, thyroid disorders, primary hyperaldosteronism, OSAS.

\section{Statistical analysis}

The distribution of variables will be assessed using the Kolmogorov-Smirnov test. To compare intergroup mean and median values, depending on variable type and distribution, we will use the T-test, the Mann-Whitney test, or the Kruskal-Wallis test for independent variables. Intergroup comparison of categorical variables will be conducted using Fisher's exact test. We will compare the variables evaluated at the beginning and during the follow-up depending on the variable type and distribution with the Paired Samples T-test and the Wilcoxon Signed Rank Test for dependent variables, with the Wilcoxon matchedpairs test for continuous and discrete variables, and with McNemar's test for categorical variables. The Pearson or Spearman's rank correlation (depending on variables distribution) will be used to assess the relationships between numerical variables. The value $P<0.05$ will be considered statistically significant.

\section{RESULTS AND DISCUSSION}

$A F$ increases the risk of dementia (hazard ratio [HR], 2.7) in patients with a history of stroke and patients with no history of stroke (HR, 1.34) [1]. This relationship is stronger in patients below 67 years of age and is related to AF duration. There are three proposed pathophysiological pathways connecting AF and brain damage: stroke and microembolization, microbleeds associated with OAC, and transient central nervous system (CNS) hypoperfusion associated with an inadequate chronotropic response. The risk of dementia depends on the $\mathrm{CHADS}_{2}$ score, regardless of warfarin treatment [2]. In subjects with AF and at least $\mathrm{CHA}_{2} \mathrm{DS}_{2}$-VASc of 2 points, the use of warfarin was associated with only a limited effect on the cognitive decline - a $20 \%$ reduction in dementia incidence. The key factor was the percentage of time during which the INR index remained within the therapeutic range. CNS vascular damage is associated with an increased risk of ischemic strokes, brain vessel microembolization, and the occurrence of microbleeds caused by supratherapeutic OAC. Furthermore, periodic hypoperfusion episodes during AF can explain the limited protective effect of OAC against dementia [3]. An average heart rate outside the $50-90 \mathrm{bpm}$ range during AF is clearly associated with decreased cognitive functions. Some studies suggest that the presence of paroxysmal AF also promotes bradycardia and subsequent CNS hypoperfusion in sinus rhythm. There are few reports of lower dementia risk in AF patients who undergo ablation [4]. However, in the EAST-AFNET4 trial, early rhythm control therapy (including 20\% early ablation and $80 \%$ pharmacological therapy in the interventional arm) did not significantly affect cognitive function [5]. The current technological progress in implantable devices makes it possible to record a high-quality EGM in the atrial channel and distinguish true AHRE artifacts. AHRE episodes are a common finding on cardiac device interrogation [6]. In the ASSERT study, during 2.5 years of follow-up of patients without previous AF history, at least one episode of AHRE lasting more than six minutes was found in about $30 \%$ of hypertensive individuals [7]. Interestingly, clinically apparent arrhythmia was eight times less frequent in this group. In the AHRE group, the probability of a stroke or peripheral embolism was twice as high as in patients with a CHADS score of 2 and without arrhythmic episodes in device memory and four times as high in patients with a CHADS score of 3 or higher. The risk of ischemic stroke in AHRE patients is significantly lower than overt AF in studies using EGM monitoring devices. First, in 2016 and subsequently, in 2020, the European Society of Cardiology guidelines on the management of atrial fibrillation recommend the use of OAC in some AHRE patients according to AHRE burden $\mathrm{CHA}_{2} \mathrm{DS}_{2}$ VASc score [8]. The recommendation concerns patients with AHRE at a frequency of $>180 \mathrm{bpm}$ (beats per minute) and lasting at least 5-6 minutes, with the AF diagnosis confirmed by visually reviewed EGM. Short ( $<1$ hour) and rare AHREs should be instead observed, excluding selected patients with a high/very high risk of stroke. The scale of potential benefit associated with the strategy of early OAC administration is being tested in two major ongoing studies: ARTESiA (Apixaban for the Reduction of Thrombo-embolism in Patients with Device-Detected Sub-Clinical Atrial Fibrillation) and NOAH AFNET 6 (Non-Vitamin K Antagonist Oral Anticoagulants in Patients with Atrial High Rate Episodes) with edoxaban $[9,10]$. The results of these studies will have crucial impor- 
tance for clinical practice and will provide missing evidence for the advisability of OAC use in AHRE. The data about the effect of AHRE on cognitive decline are limited — there are no studies that assess this issue. Our research aims to verify the suspected influence of AHRE on cognitive functions. We hypothesize that AHRE will be associated with more severe cognitive impairment in the study group. We also expect that at follow-up, the occurrence of AHRE will be associated with a faster decline of cognitive functions.

\section{Article information}

Acknowledgments: This work was supported by a grant (2.27/IV/19) from the National Institute of Cardiology in Warsaw, Poland (to SS).

Conflict of interest: MS received scientific, lecturer's or consulting fees from Abbott, Biotronik, Boehringer-Ingelheim, Boston Scientific, HammerMed, Medtronic, Pfizer, Zoll. Other authors declare no conflict of interest.

Open access: This article is available in open access under Creative Common Attribution-Non-Commercial-No Derivatives 4.0 International (CC BY-NC-ND 4.0) license, allowing to download articles and share them with others as long as they credit the authors and the publisher, but without permission to change them in any way or use them commercially. For commercial use, please contact the journal office at kardiologiapolska@ptkardio.pl.

How to cite: Sawicki S, Mierzyńska A, Krauze A, et al. Silent atrial fibrillation episodes and cognitive impairment in patients with cardiac implantable devices - protocol of the SAFE-COG ongoing observational single-center non-experimental case-control clinical study. Kardiol Pol. 2021; 79(12): 1362-1364, doi: 10.33963/KP.a2021.0162.

\section{REFERENCES}

1. De Bruijn RF, Heeringa J, Wolters FJ, et al. Association between atrial fibrillation and dementia in the general population. JAMA Neurol. 2015; 72(11): 1288-1294, doi: 10.1001/jamaneurol.2015.2161, indexed in Pubmed: 26389654

2. Graves KG, May HT, Jacobs V, et al. Atrial fibrillation incrementally increases dementia risk across all CHADS and CHADSVASc strata in patients receiving long-term warfarin. Am Heart J. 2017; 188: 93-98, doi: 10.1016/j. ahj.2017.02.026, indexed in Pubmed: 28577686.

3. Gallagher C, Lau DH, Sanders P. Reducing risk of dementia in AF: Is oral anticoagulation the key? Mayo Clin Proc. 2018; 93(2): 127-129, doi: 10.1016/j.mayocp.2017.12.017, indexed in Pubmed: 29329797.

4. Anselmino M, Gaita F, Saglietto A. Effectiveness of catheter ablation of atrial fibrillation: are we at the dawn of a new era? JThorac Dis. 2017; 9(10): 3630-3634, doi: 10.21037/jtd.2017.09.128, indexed in Pubmed: 29268360.

5. Kirchhof P, Camm AJ, Goette A, et al. Early rhythm-control therapy in patients with atrial fibrillation. N Engl J Med. 2020; 383(14): 1305-1316, doi: 10.1056/NEJMoa2019422, indexed in Pubmed: 32865375.

6. Maciąg A, Mitkowski P, Mazurek M, et al. Patient perspective and safety of remote monitoring of implantable cardioverter-defibrillators in the Polish Nationwide Multicenter Registry: the Medtronic CareLink network evaluation. Kardiol Pol. 2020; 78(11): 1115-1121, doi: 10.33963/KP.15556, indexed in Pubmed: 32794684.

7. Kaufman ES, Israel CW, Nair GM, et al. Positive predictive value of device-detected atrial high-rate episodes at different rates and durations: an analysis from ASSERT. Heart Rhythm. 2012; 9(8): 1241-1246, doi: 10.1016/j. hrthm.2012.03.017, indexed in Pubmed: 22440154.

8. Hindricks G, Potpara T, Dagres N. 12020 ESC Guidelines for the diagnosis and management of atrial fibrillation developed in collaboration with the European Association of Cardio-Thoracic Surgery (EACTS). Eur Heart J. 2021; 42(5): 373-498, doi: 10.1093/eurheartj/ehaa612, indexed in Pubmed: 32860505

9. Freedman B, Boriani G, Glotzer TV, et al. Management of atrial high-rate episodes detected by cardiac implanted electronic devices. Nat Rev Cardiol. 2017; 14(12): 701-714, doi: 10.1038/nrcardio.2017.94, indexed in Pubmed: 28682320.

10. Boriani $G$, Vitolo M. Atrial fibrillation in patients with cardiac implantable electronic devices: new perspectives with important clinical implications. Kardiol Pol. 2019; 77(12): 1119-1120, doi: 10.33963/KP.15110, indexed in Pubmed: 31855194. 